\title{
FOSS TECHNOLOGIES IN MODELLING SPATIAL ACCESSIBILITY OF PRIMARY HEALTH CARE IN MALAWI
}

\author{
Y. D. J. Phiri ${ }^{1}$, K. G. Munthali ${ }^{1,}{ }^{*}$ \\ ${ }^{1}$ University of Malawi, Chancellor College, Computer Science Department, P.O. Box 280, Zomba, Malawi - (msc-inf-10-17, \\ kmunthali)@cc.ac.mw
}

Commission IV, WG IV/4

KEY WORDS: FOSS technologies, Spatial Accessibility Modelling, Primary health care, Relational spatial analysis, PostGIS

\begin{abstract}
:
Primary health care (PHC) is the first point of contact people have with a health system. As such access to PHC services is an important factor to ensure good health of a community. While the need to provide equal and easy access to PHC is well understood, the approaches informing the decision-making process to improve the access tend to face a number of challenges in the developing world. Use of conventional Information and Communication Technologies (ICTs) comes with requisite financial costs which Free and Open Source Software (FOSS) ICT technologies have the potential to help lower among other benefits. In this study, the confluence of spatial accessibility tools provided by FOSS technologies, specifically PostgreSQL/PostGIS and QGIS, was explored to inform decision making in PHC accessibility in Zomba, Malawi. The results show that the household population (P) that is within the threshold time was 82,863 , representing $59 \%$ of all households having access to health care. The mean accessibility score for the district was 0.010 and ranged from 0.00 to 0.231 . While the findings provide, arguably, spatially objective PHC accessibility data to inform policy direction and also reveals accessibility to PHC in Malawi to be lower than reported, the study also reveals the usefulness of FOSS technologies, in the developing world. Use of FOSS facilitated incremental setup of the model thereby allowing to run the model with limited processing power. That notwithstanding, the study adds to the formal scientific research on the use of relational spatial analysis in the developing world.
\end{abstract}

\section{INTRODUCTION}

\subsection{Background}

Primary health care (PHC) is the first point of contact people have with a health system. As such access to PHC services is an important factor to ensure good health of a community. It is, therefore, essential for governments to ensure the provision of equal and easy access to PHC services to all citizens. However, uneven distribution of population, health facilities and transport infrastructure has led to spatial inequalities in accessing PHC (Kaur Khakh, Fast, Shahid, 2019; Wang, Luo, 2005). This, in turn, results in disadvantaged locations and communities having poor accessibility to needed health care facilities

While there are several definitions of access to health care, we define accessibility as an opportunity or ease with which consumers or communities can timely use appropriate services in proportion to their needs (Daniels, 1982; Peters, Garg, Bloom, Walker, Brieger, Rahman, 2008). In this context, it includes: availability, geographic accessibility, affordability, accommodation and acceptability of the health care services (Peters et al., 2008). These can be grouped into demand and supply where the former are factors influencing the ability to use health services while the latter cover aspects inherent in the health system that hinder service uptake at and by individuals, households or the community respectively (Jacobs, Bigdeli, Annear, Van Damme, 2011).
Availability is the relationship between the volume and type of existing services (and resources) to the number of clients and types of needs (Penchansky, Thomas, 1981). Geographic accessibility, on the other hand, is the relationship between the location of supply of available services, the location of clients and the characteristics of the networks linking the two locations (Vickerman, 1974). A combination of these two is what forms spatial accessibility, whose models are essential in finding the relationship between the available (supply) health care services and population (population) targeted to access the services. Spatial accessibility modelling is, therefore, an important step in policy making and improved decision making. In this case, high availability of services does not guarantee high accessibility because it depends on how close the population is to those services. On other the hand, close proximity does not guarantee high accessibility because it depends on the size of the population competing for available services (McGrail, Humphreys, 2009).

While the need to provide equal and easy access to PHC is well understood, the approaches informing the decision-making process to improve the access tend to face a number of challenges in the developing world. Attempts have been made to incorporate Information and Communication Technologies (ICTs) but the requisite financial costs, both direct and indirect, have been a constraint. The direct costs include buying or acquiring licences for the technology and the indirect include the requisite resources like availability of consistent power supply, and human capacity.

\footnotetext{
* Corresponding author
} 
Free and Open Source Software (FOSS) ICT technologies have the potential to help lower the cost barrier by reducing the cost of software, which is an important component of ICT facilities, to almost negligible levels (Tong, 2004). FOSS is downloadable without any cost and no licensing fee for each user or computer, as is for proprietary software. Upgrades of FOSS can usually be obtained in a similar way. This is in sharp contrast with proprietary software upgrades, that, normally have to be paid for.

Besides the cost benefits, there are numerous other advantages in using FOSS in health. To begin with FOSS is considered to have better reliability, performance and security. FOSS database management system of PostgreSQL/PostGIS and QGIS, used in this study, has assured high quality and compares very well with similarly proprietary applications. Pedagogically, the open philosophy of FOSS is consistent with academic freedom and the open dissemination of knowledge and information common in the academia. More importantly, the open nature of FOSS is such that it can be localized, because we have access to source code.

Given the foregoing, FOSS technologies have been touted as the twentieth century's only true innovative concept in business (Sandred, 2002) largely because it presents a very different model for organisations with regard to innovation and product development. They are a revolutionary collection of tools and processes through which individuals create, share, and apply new software and knowledge (Feller, Fitzgerald, Hissam, Lakhani 2005). FOSS prides itself on notions of peer review, building on the shoulders of giants, collaboration, openness, reputation, and standard norms of practice. FOSS is thus, a skill enabling platform that is cheaper and more adaptable to local needs (Ghosh, 2003). It allows communities to develop software that is valuable, generally reliable, globally distributed, made available for acquisition at little or no cost, and readily used in its community (Scacchi, 2004). In this study, the confluence of spatial accessibility tools provided by FOSS technologies is explored to inform decision making in primary health care accessibility in Zomba, Malawi.

\subsection{Health care delivery in Malawi}

Malawi has a population of about 17.5 million people with 84 $\%$ of it living in rural areas (NSO, 2018). Health services are delivered through government and the quasi-private Christian Health Association of Malawi (CHAM) facilities, sharing 63 and $26 \%$ of the facilities respectively with the remainder covered by private and other non-governmental facilities (WHO, 2009). Government engages these CHAM facilities through Service Level Agreements (SLAs) to deliver some health services to the communities for free. This is in an effort to improve access in terms of availability of health care services for individuals regardless of their socio-economic status more especially in rural areas where the closest facility is a CHAM facility and consequently avoid over-crowding in government facilities (Ministry of Health, 2016). It should be noted that other services are still paid for by the individual and that most private facilities and some CHAM facilities do not have any agreements with the government (Chansa, Pattnaik, 2018).

The Ministry of Health is responsible for policy making, standards setting, quality assurance, strategic planning, resource mobilization, technical support, monitoring and evaluation and international representation in Malawi. It has four main levels of delivery of health services, namely: community, primary, secondary and tertiary. Community is the lowest level focusing mainly on promotive and preventive health care provided through health surveillance assistants (HSAs) at health posts, dispensaries, and maternity clinics. At the primary level, health centres and larger community hospitals provide primary health care that includes: outpatient and inpatient services and minor procedures. The secondary level of care provides referral services to health centres and community hospitals and also provides health services to their surrounding populations. It consists of district hospitals and some CHAM hospitals of equivalent capacity. The services offered include: outpatient and inpatient services, surgical procedures like caesarean sections, herniorrhaphy and other emergency life-saving surgeries. The tertiary level consists of central hospitals to ideally provide specialist health services at the regional and national levels and referral services to district hospitals within their region. However, about $70 \%$ of the services they provide are either primary or secondary services largely due to poor gate-keeping systems (Ministry of Health, 2016). At all levels, primary health care services can be offered to the surrounding population. These different levels are linked to each other to establish a referral system that seeks to ease geographical access to and also availability of the health services.

At policy level, accessibility to health care is a radial measure where the ministry advocates that every individual should be within an $8,000 \mathrm{~m}$ radius to a health facility. The Health Sector Strategic Plan (HSSP) II, 2017-2022, thus postulates that $90 \%$ of people in Malawi have access to health care as they are within this radius. However, there are direct and indirect costs experienced to get from a household to a health facility particularly in rural areas. This is supported by the Demographic Health Survey report that indicates that about 56 $\%$ of women in rural areas struggle to access health care services citing distance as a major barrier (National Statistical Office/Malawi \& ICF, 2017). Considering that women and children are in majority such a discrepancy in policy and practice has a huge impact on the health status of the population. The discrepancies emanate from multiple factors among which is failure to incorporate critical geographic cost factors like elevation, road networks, road conditions, etc. in the estimation models. As such, while an individual could be within an $8,000 \mathrm{~m}$ radius to a health facility, the distance they might have to travel to get there may be more than $8,000 \mathrm{~m}$. Secondly, while distance can be a good indicator, the actual time taken to travel the same distance varies with geographic conditions.

Understanding that geographical accessibility to PHC is crucial and its estimation remains a challenge, this study, thus, seeks to evaluate utilisation of FOSS and geospatial routing techniques in low resource settings of Zomba, Malawi in the preparation of the data used to develop a PHC accessibility estimation model that incorporated cost spatial factors of elevation and road network distances.

\subsection{Study area}

Zomba is one of the 28 districts in Malawi located in the southern region and belongs to the south eastern zone of the health administration. It has a population of about 851,737 people (NSO, 2018), $88 \%$ of which live in rural areas. The district has 554 enumeration areas with an average of 233 households per enumeration area suggesting an average of 4 people per household (NSO, 2018). Zomba is divided into Traditional Authorities (TA) governed by local leaders and each TA is further subdivided into villages to form a hierarchy of 
local leadership. There is no organised public transport in Zomba and though the road network coverage is good, most of the roads are not paved limiting their utilisation with motorised transportation. The study area is presented in Figure 1.

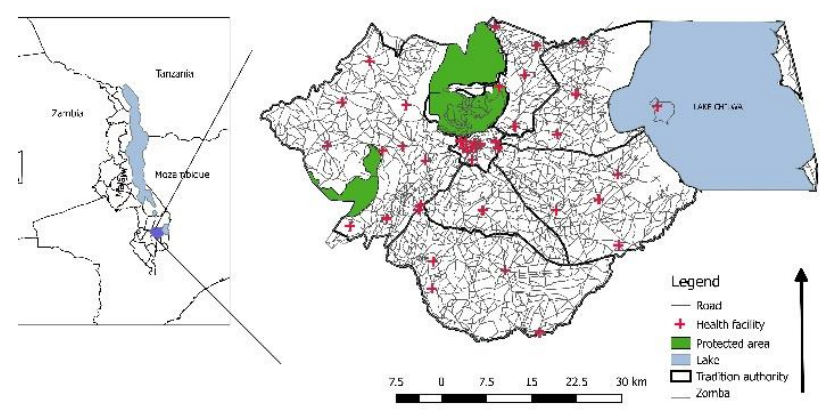

Figure 1 Study area

\section{METHODOLOGY}

\subsection{Data Collection and Preparation}

To develop the estimation model data was collected using both primary and secondary sources. The study population consist of all health facilities under Zomba District Health Office, households in rural and urban areas accessing health services. Currently, there are 45 health facilities registered under Zomba District Health Office (DHO) that include: a central hospital, a rural hospital, 10 CHAM facilities, 28 health centres and 5 dispensaries.

Primary data was collected using interview and questionnaires. It collected data on methods used to determine accessibility, plan for a new facility, capacity of different type of health facilities and the number of personnel offering the services at the District Health Office (DHO) and facility levels. To understand the perspective of households on health care access, a questionnaire was administered to collect data about the location of the household, mode of transport used to get to a facility, the facilities where the household go for medical attention, and any geographic factors affecting the households' access to health care. Secondary data included household data, road network, and mode of transport, Digital Elevation Model (DEM), health facility names and location of facilities from Health Management Information System, mode of client transport to the clinic, services offered at health facilities and number of personnel offering the services. 2008 census data provided the population in the area and number of households indicating the demand for services at the facility. The health facility positions were linked with the number of health workers at the facilities and their standard target population.

While both data sets were relevant for the model, the methodology concentrates on the spatial data and how it was prepared for the model using FOSS technologies primarily PostgreSQL/PostGIS for the spatial accessibility analysis and QGIS for visualisations. The rest of the analyses and synthesis of the results built on the flexibility and power of structured query language (SQL) provided through PostgreSQL/PostGIS.

\subsection{Data processing methods}

The procedure to process and prepare the data for the spatial modelling is as shown in Figure 2 parts $\mathbf{A}$ and B. Despite increasing the computational overheads, the road network was exploded into smaller road segments. This was necessitated by the fact that this improved the accuracy of tracing paths to the households and facilities, on one hand, and the slope calculation on the other. The function used was explode lines in QGIS. These were then converted into edges and vertices using the pg_routing create topology function in PostgreSQL/PostGIS. This function generates a spatially conformant topology of the road network that optimises storage space, ensures connectedness and provides explicit spatial relationships. Thereafter, the vertices were layered over the DEM and the elevation values of each vertex on the road was extracted.

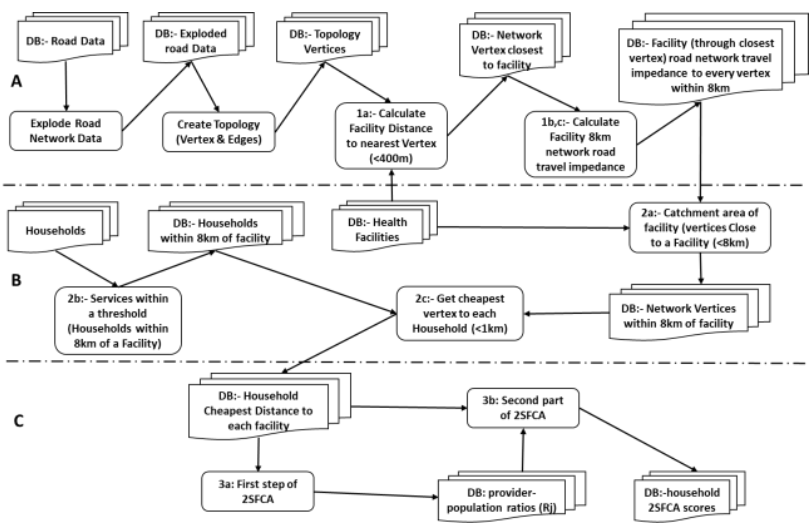

Figure $2 \mathbf{A}$ and $\mathbf{B}$, Data processing; $\mathbf{C}$, spatial accessibility modelling

The next step was to identify a vertex on the road network closest to every facility. This involved calculating the distances from each facility to every vertex and then finding the smallest distance for that facility. The former used the optimised st distance function while the latter used the minimum aggregate function in SQL per facility. Thereafter, the network travel cost from a facility, using the identified closest vertex on the road, to every vertex on the road network for vertices within an $8000 \mathrm{~m}$ radius was calculated. This included identifying the travel path on the network using the pgr_dijkstraCost function in PostgreSQL/PostGIS and an impedance factor of slope derived from elevation. The Dijkstra algorithm, despite having a higher order of magnitude in terms of complexity than the astar, was preferred as it has no inherent limit in its search space. The overall travel path cost was obtained as follows:

$$
\text { Travel Path Cost }=\sum_{\mathrm{i}=0}^{\mathrm{n}}\left(\left(\left[\frac{a_{\mathrm{ina}}-{\omega_{i}}_{\mathrm{i}}}{x}\right]+1\right) x\right)
$$

where $n$ is the number of vertices forming the path $a_{i}$ represents elevation at vertex $\tilde{i}$ on the path $x$ is the length of the road segment on the path; and 1 translates negative slope value (descending) to an accelerating factor by reducing the travel cost from $a_{i+1}$ to $a_{\mathrm{i}}$.

To determine household cheapest travel cost to a facility; the model determined the households within an 8,000 m radius of a facility on one hand and network vertices within $8,000 \mathrm{~m}$ travel cost of a facility. Then, for each household in the former, the model identified the cheapest vertex closest to household within a $1,000 \mathrm{~m}$ radius. The $1,000 \mathrm{~m}$ radius was justified for two reasons: i) reduce the search space and improve computational processing; ii) avoid reducing the model to straight line distance. 


\subsection{Spatial modelling and analysis}

Spatial accessibility was done using the Two-Step Floating Catchment Area (2SFCA) method with an addition of the Gaussian continuous decaying function $f\left(d_{i j}\right)$. The continuous decay function removes the assumption that all the households living within a specified threshold have the same accessibility index score (Luo, Qi, 2009).

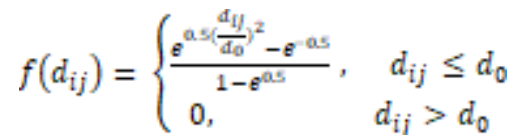

where $f\left(d_{i j}\right)$ is impedance weighting $d_{i j}$ is the time between point $\tilde{i}$ and $j$; and $d_{0}$ is the time threshold

The 2SFCA creates two floats (catchments): service catchment and population catchment within a specified time of travel. Each step creates an area of coverage called catchment that are floated on each other (Wang, Luo, 2005). The first is for each service (S) located at $\tilde{f}$, find all household population $(\mathrm{P})$ that is within a threshold time $\left(d_{0}\right)$ located at $k$ (implying catchment area of health centre), calculate the population-to-provider ratio $R_{j}$ represented as:

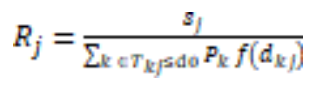

The second step is, for each population, find all services that fall within a threshold time $\left(\mathrm{d}_{0}\right)$ accessible at location $i$ and sum the population-to-provider ratios from step 1 represented by:

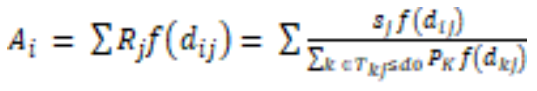

Where $A_{i}$ represents the accessibility of household at location $\tilde{i}$.

$P_{k}$ is the population at location $k$;

$S_{j}$ is the service capacity at location $f$;

$d_{i j}$ is the travel distance between $k$ and $j$ expressed in terms of time;

$R_{j}$ is the service to population ratio at location $j$ that falls within the catchment $\left(d_{k j} \leq \mathrm{d}_{0}\right)$.

A larger value of $A_{\tilde{T}}$ indicates the better accessibility to services at that household location

\section{RESULTS AND DISCUSSION}

The road network vertex closest to a facility result contained the distance from the facility to the vertex corresponding to the shortest distance. The distances ranged between 6 to $338 \mathrm{~m}$ with an average of $113 \mathrm{~m}$ for the 41 health facilities in the study area. To reduce the search space, and hence reduce computation time, the query only checked for vertices within a radius of $400 \mathrm{~m}$. This radius is the maximum distance for all facilities to a road rounded off to the next higher 100. Figure 3 illustrates the resulting implementation narrowed down to Likangala Health Centre.

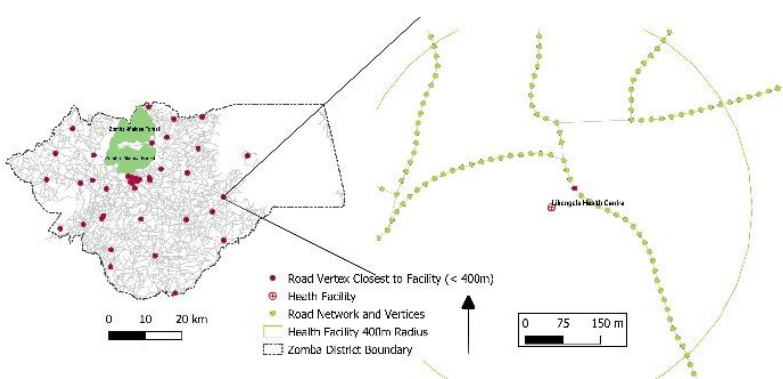

Figure 3 Road vertex closest to Likangala Health Centre

The network travel cost from a facility to every vertex on the road network, calculated from the vertex closest to the facility, averaged $5224 \mathrm{~m}$ with a minimum of $19.5 \mathrm{~m}$ and maximum of $8000 \mathrm{~m}$. Factoring in the slope, the travel cost averaged $5238 \mathrm{~m}$ with a minimum of $16.9 \mathrm{~m}$ and maximum of $9042.5 \mathrm{~m}$. Figure 4 shows the network travel cost compared against the straight-line distances for each of the facilities.

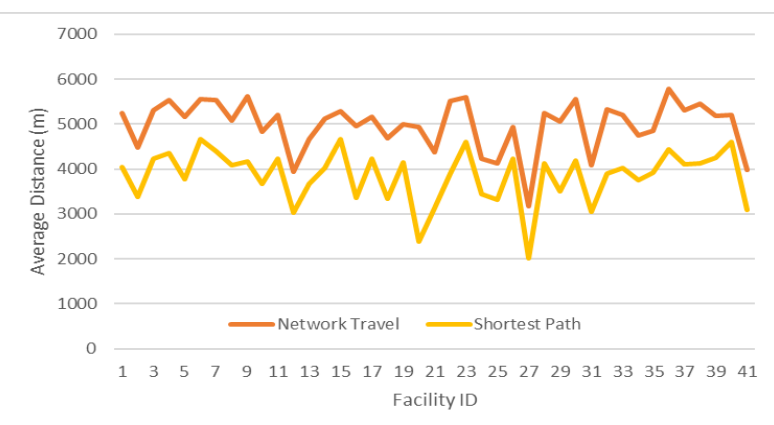

Figure 4 Average travel distance for the facilities

Further, the national policy indicates that households within an $8000 \mathrm{~m}$ radius to a facility could be regarded as having access to health care even when the travel distance could be greater than $8000 \mathrm{~m}$. In Figure 5, a total of 6,140 households are between the 6000 to $8000 \mathrm{~m}$ of Namikango Health Centre and as such, by the national policy, they have access to health care at Namikango. However, $4,331(71 \%)$ of these households have travel distances greater than $8000 \mathrm{~m}$ and consequently, for policy and planning purposes, the results suggest these to, at best, be considered not to have access to health care through Namikango.

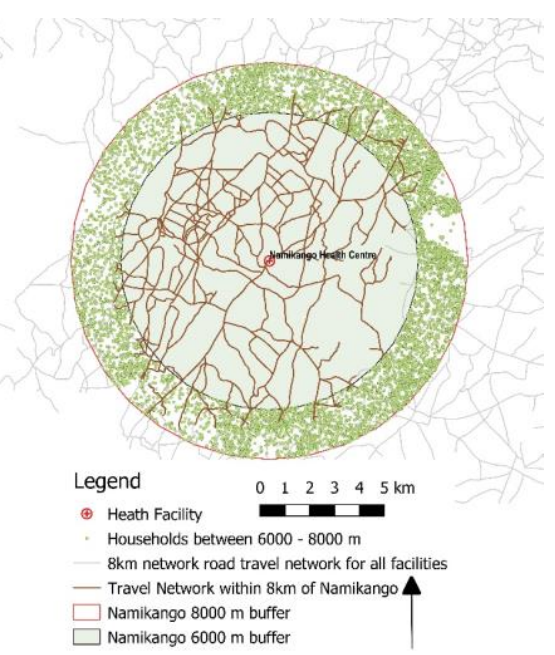

Figure $58000 \mathrm{~m}$ Travel network for a facility (Namikango) 
Although it is intuitive that the travel distance would be comparatively higher than the straight-line distance, for the rural areas the straight line gives a more realistic representation especially for households within the vicinity of the facility. In such instances the model overestimated the distances. In fact, for households on the same side of the road as the health facility and within, say a kilometre of that facility, accessing the facility using the road vertex closest to the facility was counter intuitive (see Figure 6). With walking as the mode of transportation, such households would rather use uncharted routes like footpaths or trails to get to the facility than use the road. This would not hold for farther households, as eventually they would have to cut through to a road to get to the facility. In spite of this limitation, the model opted for the assessment based on the travel distance through vertex for three reasons: i) very few of the households are within the vicinity of the facility; ii) if a significant number of the households circumvent the vertex, the model reduces to a straight line distance assessment; and iii) the middle ground of comparing the travel distance against the straight line distance and opting for the smaller value was going to be computationally expensive.

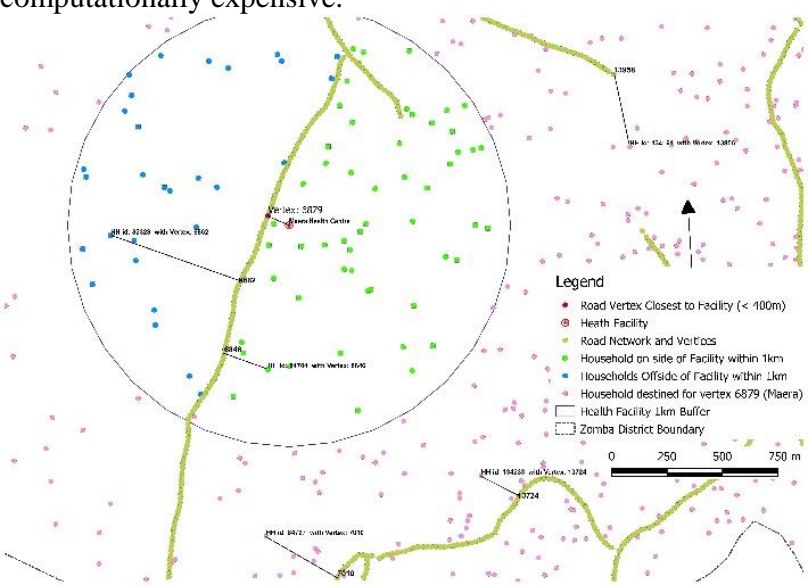

Figure 6 Straight line versus travel distance conundrum for households within the vicinity of a facility

Notwithstanding the $8000 \mathrm{~m}$ shortest and travel distances, the World Health Organisation (WHO) recommends that accessibility be determined using travel time rather than distance (WHO, 2009) as the former not only takes care of the latter but also inherently accounts for mode of transport. The study adopted a 60-minute maximum threshold to assess whether a household has access to health care as implemented by other researchers (dos Anjos Luis, Cabral, 2016; Huerta Munoz, Källestål, 2012; Wang, Luo, 2005; Schuurman, Bérubé, Crooks, 2010). With walking as the adopted mode of transport in the study area, and averaged at $5 \mathrm{~km}$ per hour, accessibility in terms of travel distance was capped at $5,000 \mathrm{~m}$. As shown in Table $1,61 \%$ of the households travel less than $5,000 \mathrm{~m}$ to access health care.

Table 1 Health care accessibility for Zomba based on distance

\begin{tabular}{|c|c|c|c|c|c|c|}
\hline \multirow[b]{2}{*}{ Distance } & \multicolumn{2}{|c|}{ Urban } & \multicolumn{2}{|l|}{ Rural } & \multicolumn{2}{|l|}{ Overall } \\
\hline & Households & $\%$ & Households & $\%$ & Households & $\%$ \\
\hline$<2 \mathrm{~km}$ & 12,461 & 69.50 & 13,286 & 11 & 25,747 & 18 \\
\hline 2 to 4 & 5,352 & & 33,077 & 27 & 38,429 & 28 \\
\hline $\mathrm{km}$ & & 29.85 & & & & \\
\hline $\begin{array}{l}4 \text { to } 5 \\
\mathrm{~km}\end{array}$ & 103 & 0.57 & 21,102 & 17 & 21,205 & 15 \\
\hline$>5 \mathrm{~km}$ & 14 & 0.08 & 54,250 & 45 & 54,264 & 39 \\
\hline Total & 17,930 & & 121,715 & & 139,645 & \\
\hline
\end{tabular}

Therefore, using the WHO-recommended threshold time of 60 minutes, the household population $(\mathrm{P})$ that is within the threshold time was 82,863 , representing $59 \%$ of all households having access to health care. Correspondingly, as shown in Table 2, $99.9 \%$ of the household's in the urban areas have access to health care, with $51 \%$ being within $20-$ minute distance, as opposed to $53 \%$ in the rural where only $13 \%$ are within 20-minute distance. Figure 7 illustrates the situation for the study area in terms of distances.

Table 2 Health care accessibility for Zomba based on time

\begin{tabular}{lr|rr|r|r|r}
\hline Travel Time & \multicolumn{2}{c|}{ Urban } & \multicolumn{2}{c|}{ Rural } & \multicolumn{2}{c}{ Overall } \\
\cline { 2 - 7 } (minutes) & Households & \% & Households & \% & Households & \% \\
\hline$<=20$ & 9,129 & 51 & 8,308 & 13 & 17,437 & 21 \\
20 to 40 & 7,836 & 44 & 23,259 & 36 & 31,095 & 38 \\
40 to 60 & 950 & 5 & 33,381 & 51 & 34,331 & 41 \\
\hline Total & $\mathbf{1 7 , 9 1 5}$ & $\mathbf{6 4 , 9 4 8}$ & $\mathbf{8 2 , 8 6 3}$ & \\
\hline
\end{tabular}

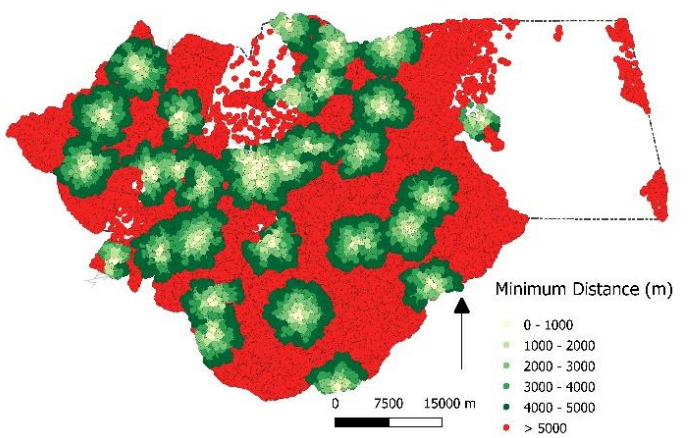

Figure 7 Health care accessibility for households in Zomba.

For all households the accessibility score was calculated using 2SFCA with a threshold of 60 minutes. The higher the score the better the accessibility. For household that their travel time to the nearest facility was above 60 minutes were considered underserved i.e. having poor access. The 2SFCA score starts from 0 growing boundlessly with 0 indicating households not within the 60-minute threshold. Higher scores indicate access within 60 minutes and to multiple facilities or multiple services within an hour or facility respectively. The mean accessibility score for the district was 0.010 and ranged from 0.00 to 0.231 (see Figure 8$)$. The highest accessibility score $(>=0.2)$ was located around Chisi Health centre, largely because the area is an island with relatively low population.

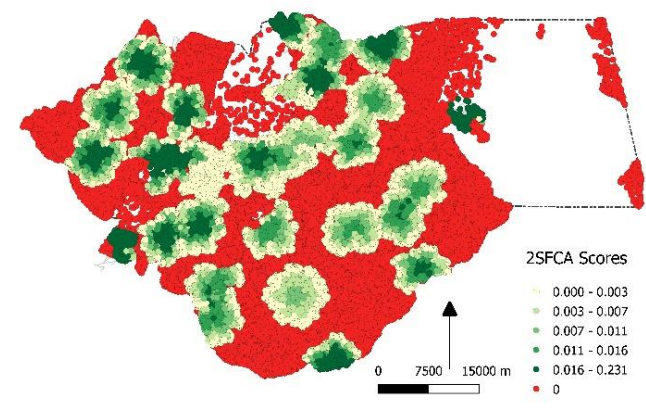

Figure 8 Distribution of accessibility scores for Zomba 
Most households in the urban area have good accessibility scores, with all households in the urban area having access to at least five facilities within a 60-minute travel distance as opposed to 43.6 and $34.4 \%$ in the rural areas who have access at most one and two facilities respectively within a 60 -minute travel distance. Table 3 provides more details. The model also found out that the households in the district boundaries have better accessibility scores e.g. around Ngwerero, Maera and Namasalima Health Centres. This is largely due to the fact a significant population of the households serviced by these hospitals from the neighbouring districts were not included in this study.

Table 3 Number of facilities accessed by households

\begin{tabular}{rr|r|r|r|r|r}
\hline \multirow{2}{*}{$\begin{array}{c}\text { Facility } \\
\text { count }\end{array}$} & \multicolumn{2}{c|}{ Urban } & \multicolumn{2}{c|}{ Rural } & \multicolumn{2}{c}{ Overall } \\
\cline { 2 - 7 } & Households & \multicolumn{1}{c}{$\%$} & Households & \% & Households & \% \\
\hline 1 & - & 0 & 53,051 & 43.6 & 53,051 & 43.6 \\
2 & - & 0 & 41,881 & 34.4 & 41,881 & 34.4 \\
3 & - & 0 & 14,124 & 11.6 & 14,124 & 11.6 \\
4 & - & 0 & 4,050 & 3.33 & 4,050 & 3.33 \\
5 & - & 0 & 1,147 & 0.94 & 1,147 & 0.94 \\
$>5$ & 17,930 & 100 & 7,462 & 6.13 & 25,392 & 20.9 \\
\hline Total & $\mathbf{1 7 , 9 3 0}$ & $\mathbf{1 2 1 , 7 1 5}$ & $\mathbf{1 3 9 , 6 4 5}$ & \\
\hline
\end{tabular}

Since Malawi is aiming at universal health coverage through increased equitable access to and improve the quality of health care services, sound and formal assessments of access to health care, service coverage and analysing underserved population are important. It is not surprising then that, in the absence of any formal analysis tools, critical decisions tend to be made more on political rather than pragmatic considerations, with far less optimal results (Rahman, Smith, 2000). This evaluation of spatial accessibility to health services offers critical information for public policy planning and service provision as it allows for the identification of areas with lower or higher access to resources. Therefore, having reliable information to determine accessibility of the current health system, what services should be added and what challenges people meet to access these services is important for decision making to achieve universal health coverage.

While the findings provide, arguably, spatially objective PHC accessibility data to inform policy direction and also reveals accessibility to PHC in Malawi to be lower than reported, the study also reveals the usefulness of FOSS technologies, specifically PostgreSQL/PostGIS and QGIS, in the developing world. With increasing licencing costs and repeated upgrade cycles for proprietary software, FOSS technologies have provided a cost-effective solution to the community, ministry and government. This study substantiates reports that communities can save a lot of money if they adopt FOSS (Tiemann, 2009a, 2009b). More so in the sub-Saharan region when ICTs, and FOSS specifically, are taken to be "enablers" for economic growth. The implementation of the model using FOSS technologies facilitated incremental setup of the model thereby allowing to run the model with limited processing power of personal computers. That notwithstanding, the study adds to the formal scientific research on the use of relational spatial analysis in the developing world through the use PostgreSQL/PostGIS and QGIS. With the persistent data approach of a database management system, the use of PostgreSQL allowed the model to run successfully with intermittent power supply. The combination of being able to build the model incrementally and at the same being able to continue in the case of power cuts cannot be overemphasised for developing countries. Without FOSS, such modelling becomes very difficult to implement at larger scales.

\section{CONCLUSION}

The results indicate that only $59 \%$ of the households have access to health care within the recommended travel threshold of 60 minutes. This is lower than $61 \%$ indicated by travel distance in Table 1 and $90 \%$ as provided for by the ministry based on straight-line distance. The result of the study agrees with the DHS report that indicated an accessibility of $56 \%$ for Malawi. Besides the evaluation of PHC accessibility, the application of FOSS tools in this study, strengthens the case of their relevance in low resource contexts where computation power is limited and power supply intermittent, making it difficult to run models that span multiple days. Using PostgreSQL/PostGIS for relational spatial analysis modelling made it possible to resume modelling after power outages. Thus, the study has successfully demonstrated utility of relational spatial analysis using FOSS technologies in low resource settings of the developing world. With improving computational capabilities such models can be used for national benchmarking of accessibility and hence provide concrete data on the provision of PHC and other applications.

\section{REFERENCES}

Chansa, C., Pattnaik, A., 2018: Expanding health care provision in a low-income country: the experience of Malawi (English). Universal health coverage study series; no. 34. Washington, D.C.: World Bank Group.

Daniels, N., 1982: Equity of Access to Health Care: Some Conceptual and Ethical Issues. The Milbank Memorial Fund Quarterly. Health and Society, 60(1), 51-81. doi.org/10.2307/3349700

dos Anjos Luis, A., Cabral, P., 2016: Geographic accessibility to primary healthcare centers in Mozambique. International Journal for Equity in Health, 15(1), 173.

Feller, J., Fitzgerald, B., Hissam, S.A., Lakhani, K.R., 2005: Perspectives on Free and Open Source Software. The MIT Press.

Ghosh, R. A., 2003: Licence fees and GDP per capita: The case for open source in developing countries. First Monday, 8. doi.org/10.5210/fm.v8i12.1103

Jacobs, B., Bigdeli, M., Annear, P. L., Van Damme, W., 2011: Addressing access barriers to health services: an analytical framework for selecting appropriate interventions in lowincome Asian countries. Health Policy and Planning, 27(4), 288-300.

Kaur Khakh, A., Fast, V., Shahid, R., 2019: Spatial Accessibility to Primary Healthcare Services by Multimodal Means of Travel: Synthesis and Case Study in the City of Calgary. International Journal of Environmental Research and Public Health, 16(2), 170. doi.org/10.3390/ijerph16020170

Luo, W., Qi, Y., 2009: An enhanced two-step floating catchment area (E2SFCA) method for measuring spatial accessibility to primary care physicians. Health \& Place, 15(4), 1100-1107. doi.org/10.1016/j.healthplace.2009.06.002

McGrail, M. R., Humphreys, J. S., 2009: The index of rural access: an innovative integrated approach for measuring primary care access. BMC Health Services Research, 9(1), 124. doi.org/10.1186/1472-6963-9-124

Ministry of Health, 2016: Universal Access. http://www.health.gov.mw/index.php/77-demo/80-joomladevelopment. (18 March 2018) 
National Statistical Office/Malawi and ICF. 2017. Malawi Demographic and Health Survey 2015-16. Zomba, Malawi: National Statistical Office and ICF. FR319

NSO, 2018: 2018 Malawi Population and Housing Census. http://www.nsomalawi.mw/index.php?option=com_content\&vi ew=article\&id=226\&Itemid=6 (11 March 2019).

Penchansky, R., Thomas, J. W., 1981: The Concept of Access: Definition and Relationship to Consumer Satisfaction. Medical Care, 19(2), 127-140.

Peters, D. H., Garg, A., Bloom, G., Walker, D. G., Brieger, W. R., Rahman, M. H., 2008: Poverty and access to health care in developing countries. Annals of the New York Academy of Sciences, 1136, 161-171. doi.org/10.1196/annals.1425.011

Rahman, S., Smith, D. K., 2000): Use of location-allocation models in health service development planning in developing nations. European Journal of Operational Research, 123(3), 437-452. doi.org/10.1016/S0377-2217(99)00289-1

Sandred, J., 2002: Managing open source projects: A Wiley tech brief (Vol. 18). John Wiley \& Sons.

Scacchi, W., 2004: Free and open source development practices in the game community. IEEE Software, 21(1), 59-66. doi.org/10.1109/MS.2004.1259221

Schuurman, N., Bérubé, M., Crooks, V. A., 2010: Measuring potential spatial access to primary health care physicians using a modified gravity model. The Canadian Geographer / Le Géographe Canadien, 54(1), 29-45. doi.org/10.1111/j.15410064.2009.00301.x

Tiemann, M., 2009a: How open source software can save the ICT industry one trillion dollars per year. https://opensource.org/files/OSS-2009.pdf. (3 march 2019)

Tiemann, M., 2009b: Open Source Software Can Save India \$2 BN/year--At least! | Open Source Initiative. https://opensource.org/node/467 (14 march 2019)

Tong, T.W., 2004: Free/Open Source Software Education. ISBN: 81-8147-565-8, ELSEVIER New Delhi, India

Vickerman, R. W., 1974: Accessibility, attraction, and potential: a review of some concepts and their use in determining mobility. Environment and Planning A, 6(6), 675691.

Wang, F., Luo, W., 2005: Assessing spatial and nonspatial factors for healthcare access: towards an integrated approach to defining health professional shortage areas. Health \& Place, 11(2), 131-146.

WHO, 2009: WHO Country Cooperation Strategy 2008-2013 Malawi. ISBN: 9789290231134 